\title{
Wavelet Analysis in EPR Spectroscopy
}

\author{
A. Drzewiecki* And P.B. ScZAniecki \\ Institute of Physics, University of Zielona Góra \\ Szafrana 4a, 65-516 Zielona Góra, Poland
}

\begin{abstract}
Possibility of application of wavelet analysis in EPR spectroscopy is investigated. Basic mathematics of the method is summarized, with emphasis on the relation between EPR experiment and physical interpretation of wavelet transform. The fact that first derivative of the Lorentzian function can be used as a base wavelet is employed and the specific rules of the interpretation of the final wavelet synthesis are identified. Analysis of a sample EPR spectrum of X-ray irradiated guanidinium aluminium sulphate hexahydrate crystal is given as an example. Set of parameters resulting from the wavelet analysis is subsequently used to reconstruct/synthesize the original EPR spectrum. The method involves an opportunity of eliminating unwanted components of the multicomponent spectra. Generally, the method may supplement other means of data analysis, in particular, in magnetic resonance.
\end{abstract}

PACS numbers: 07.05.Kf, 76.30.Rn, 82.50.Kx

\section{Introduction}

Wavelet analysis is a modern and popular method of data analysis. Applications of this method are surprisingly wide: from seismology through cardiology, it ends up with digital image compression. The basic keyword is multiresolution analysis - it makes it so widely used.

Typical EPR spectrum is a sum of components, originating from paramagnetic centers of the studied sample. The line shape of EPR lines of particular paramagnetic center is usually Gaussian or Lorentzian function, they differ in the resonance field, their amplitude, and line widths. We want to obtain magnitudes of these parameters.

Multicomponent EPR spectra are usually recorded as derivatives of paramagnetic absorption. In order to find resonance fields of particular centers, we

*corresponding author; e-mail: A.Drzewiecki@if.uz.zgora.pl 
need to know where the derivatives are zero. But in a real spectrum it is difficult to determine these points, especially when the lines are too close to each other, relative to their line widths, or if line widths significantly differ - even worse, when their amplitudes differ significantly. If one of these facts occurs, the typical method of determination of resonance fields - differentiating the recorded spectrum, and finding maxima of derivative - fails.

Wavelet analysis of EPR spectrum of guanidinium aluminium sulphate hexahydrate (GASH) crystals irradiated with X-ray is examined and discussed. The spectrum is complex: there are three equal lines, originating from $\mathrm{SO}_{4}^{3-}$ ions [1], but the measured spectrum exhibits a broad line of large amplitude, which obstructs the analysis of the other lines. Wavelet analysis of the spectrum can allow to distinguish lines with different line widths, which makes possible to extract and remove the broad line from the spectrum and analyze the remaining components, however the removal is neither exact nor automatic. The conclusion is that the method is useful as a supporting tool in the interpretation of these types of EPR spectra.

\section{Basics of wavelet analysis}

Wavelet analysis of a signal provides information on both: time and frequency domains. Of course, the two-dimensional analysis of one-dimensional data set must be redundant, but the additional information which we obtain is the argument for wide application of this method.

Information which we obtain in the two dimensions is not independent. Localization in time and frequency is limited by Heisenberg's rule of uncertainty. However, within this limit we obtain information on both dimensions at once. Alternatively, if we have a signal, we know exact value of it in a given time, but we know nothing about the frequency. Of course, we can make the Fourier transform of this signal, and then we will know all about frequencies of the signal, but we do not know the values of signal at a given time. Wavelet analysis offers an information on both of these dimensions simultaneously [2].

From mathematical point of view, the wavelet transformation is defined as follows:

$$
\Psi(t, s)=\int_{-\infty}^{\infty} f(x) \frac{1}{\sqrt{s}} \varphi\left(\frac{x-t}{s}\right) \mathrm{d} x,
$$

where $f(x)$ is the analyzed function, $\varphi$ is a function called base wavelet, and $t$ and $s$ are parameters, controlling the processes called translation and scaling, respectively. Interpretation of this integral is simple: the obtained value is a measure of similarity of function $f(x)$ and function $\varphi$, translated $t$ units to the right on $x$-axis and then stretched $s$ times [3]. Both functions $f(x)$ and $\varphi$ must be square integrable. 
Proper base wavelet must satisfy the following conditions:

1. mean value of base wavelet must be equal to zero

$$
\int_{-\infty}^{\infty} \varphi(x) \mathrm{d} x=0
$$

2. base wavelet must be square integrable

$$
\int_{-\infty}^{\infty}|\varphi(x)|^{2} \mathrm{~d} x<\infty
$$

3. the following integral must have a finite value:

$$
C_{\varphi}=\int_{-\infty}^{\infty} \frac{|\tilde{\varphi}(\omega)|}{|\omega|} \mathrm{d} \omega<\infty,
$$

where numerator is the modulus of the Fourier transform of $\varphi$.

There are physical arguments to adopt Lorentzian or Gaussian function as EPR absorption line shapes. In the magnetic resonance context, these functions must be augmented with these parameters:

- $A$ - amplitude of the line, a multiplicative factor;

- $B_{0}$ - resonance field, as a subtractive factor to magnetic field $B$;

- $\Delta B$ - half-width at $1 / 2$ amplitude for the Lorentzian shape (for Gaussian function this definition is similar, except that half-width must read at $1 / \mathrm{e} \approx$ $1 / 2.78$ amplitude).

For Lorentzian function (for Gaussian function reasoning is the same)

$$
f(x)_{\mathrm{L}}=\frac{1}{1+x^{2}}
$$

inserting EPR parameters gives the following function:

$$
f_{A, B_{0}, \Delta B}(B)_{\mathrm{L}}=A f\left(\frac{B-B_{0}}{\Delta B}\right)_{\mathrm{L}}=\frac{A}{1+\left(\frac{B-B_{0}}{\Delta B}\right)^{2}} .
$$

Lorentzian function is used in the expression above in the same form as the base wavelet in the wavelet transform, except multiplication by $A$. The role of translation parameter $t$ is taken by the resonance field $B_{0}$ and the role of scaling parameter $s$ goes to the line width, respectively.

The basic problem is that neither Gaussian nor Lorentzian function can be base wavelets - they have nonzero mean value. Their first derivatives however, satisfy all the requirements (Eqs. (2.2)-( 2.4)). As mentioned above, EPR spectra are usually recorded as derivatives of EPR absorption, which is advantageous for the method. First derivative of Lorentzian line has the form (for derivative of Gaussian function the multiplying factor is the same) 


$$
f_{A, B_{0}, \Delta B}^{\prime}(B)_{\mathrm{L}}=\frac{A}{\Delta B} f^{\prime}\left(\frac{B-B_{0}}{\Delta B}\right)_{\mathrm{L}}=-\frac{2 \frac{A}{\Delta B} \frac{B-B_{0}}{\Delta B}}{\left[1+\left(\frac{B-B_{0}}{\Delta B}\right)^{2}\right]^{2}} .
$$

Again the form of this equation is the same as base wavelet in wavelet transform, except for the multiplying factor. This leads to key conclusion: if recorded spectrum is linear combination (at least approximately) of derivatives of Lorentzian functions (Eq. (2.7)), we can seek them by wavelet analysis: set of translated and scaled base wavelets is regarded in the scalar product, in wavelet transformation as something similar to "base in linear space".

We need to examine the properties of wavelet transformations with the selected base wavelet. Base wavelet is the basic element sought by the transformation. With the choice of normalized Lorentzian wavelet

$$
\varphi\left(\frac{x-t}{s}\right)=-\frac{4}{\sqrt{\pi}} \frac{\frac{x-t}{s}}{\left[1+\left(\frac{x-t}{s}\right)^{2}\right]^{2}}
$$

the transformation has the form

$$
\begin{gathered}
\Psi(t, s)=\int_{-\infty}^{\infty} f_{A, B_{0}, \Delta B}^{\prime}(x)_{\mathrm{L}} \frac{1}{\sqrt{s}} \varphi\left(\frac{x-t}{s}\right) \mathrm{d} x \\
=-\frac{4 A \sqrt{\pi} \Delta B s^{\frac{3}{2}}}{(s+\Delta B)^{3}} \frac{3\left(\frac{t-B_{0}}{s+\Delta B}\right)^{2}-1}{\left[\left(\frac{t-B_{0}}{s+\Delta B}\right)^{2}+1\right]^{3}} .
\end{gathered}
$$

The properties of this transformation are:

- with respect to the variable $t$, wavelet transformation of Lorentzian line has the shape of second derivative of Lorentzian function (Fig. 1);

- treated as a function of two variables (Eq. (2.9)) has one maximum at $\left(t=B_{0}, s=\Delta B\right)$. The coordinates of the maximum yield two of the three basic parameters of the analyzed spectral line;

- knowing the coordinates of the maximum and its magnitude, one may also compute the amplitude of the line from the following formula:

$$
A=\left.2 \sqrt{\frac{\Delta B}{\pi}} \Psi(t, s)\right|_{t=B_{0}, s=\Delta B} .
$$

- properties of the transformation with respect to variable $s$ causes a suppression of these components whose line widths significantly differ from the local value of $s$. This mechanism may help to filter a line of a specified width;

- transformation of a single line consists of three regions: central positive part that includes the global maximum and two negative parts on left and right side, their separation is a linear function of $s$. 


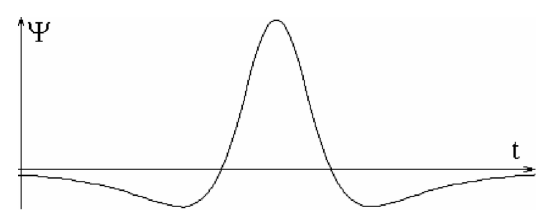

Fig. 1. Shape of wavelet transformation with respect to the variable $t$. The horizontal line marks zero level.

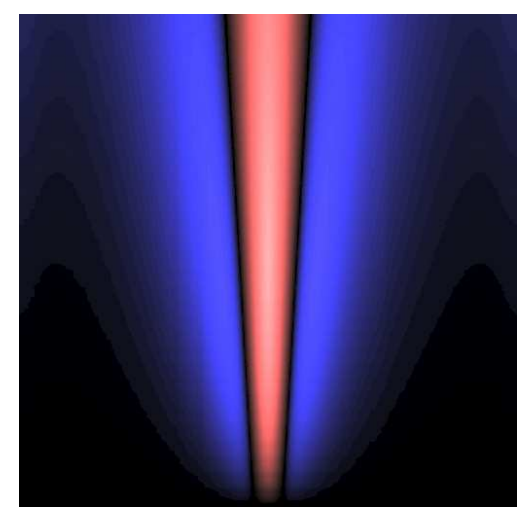

Fig. 2. Results of wavelet analysis of a sample Lorentzian derivative obtained with numerical program "Analizator"*. Horizontal and vertical axis denote $t$ and $s$, respectively.

Each line in the complex spectrum generates additive ingredients in the form of above function to summary wavelet analysis. In this particular picture the patterns from left to right correspond to negative-positive-negative values, reflecting the shape of the function in Fig. 2.

\section{Wavelet analysis - an example}

Guanidinium aluminium sulphate hexahydrate is a ferroelectric with space group $C_{3 v}^{2}(P 31 m)$. The crystal formula is $\mathrm{C}\left(\mathrm{NH}_{2}\right)_{3} \mathrm{Al}\left(\mathrm{H}_{2} \mathrm{O}\right)_{6}\left(\mathrm{SO}_{4}\right)_{2}$ : three nearly planar guanidinium groups are sandwiched between the hexaquo-metal octahedra. Exposing the crystal to X-ray $K_{\alpha}$ radiation for 2 hours produces paramagnetic, anisotropic centers whose EPR will be used as an example of the application of the wavelet analysis. Three overlapping components (Fig. 3) can be distinguished, all in the vicinity of free radical $g$-value [4].

Wavelet analysis of this EPR spectrum (Fig. 3) is presented below (Fig. 4a). The red-colored regions represent the positive magnitudes and the blue-colored regions represent the negative values of the analysis (Eq. (2.9)).

Horizontal cross-section of the above pictures reveals that the analysis has a maximum at $\left(t_{1}, s_{1}\right)$. Large value of $s_{1}$ gives evidence of a broad line. On the

${ }^{*}$ The program, written in $\mathrm{C}++$, is available from the primary author upon request. 


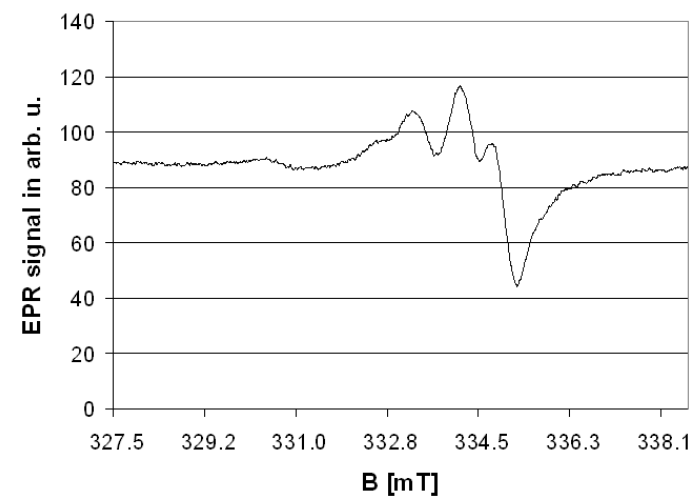

Fig. 3. Recorded EPR spectrum of X-ray irradiated GASH crystal.
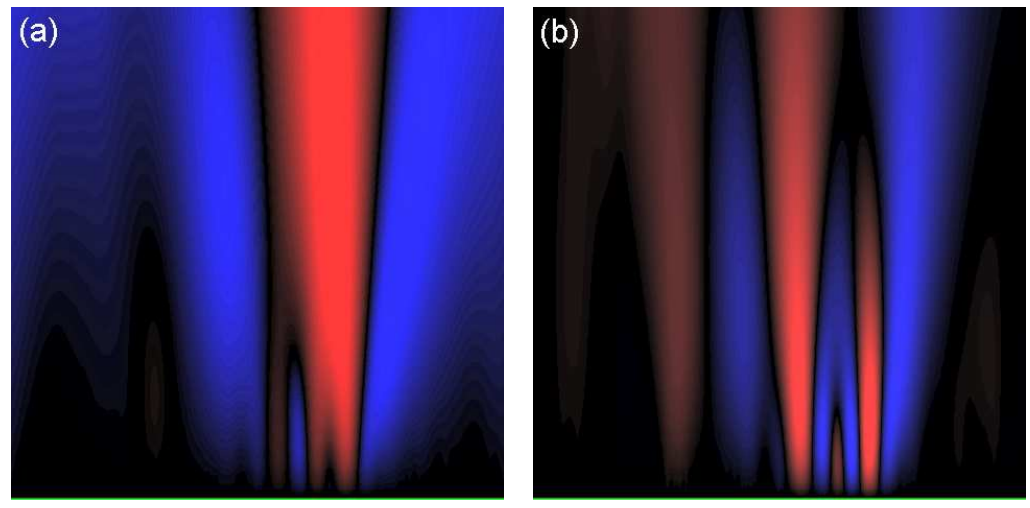

Fig. 4. (a) Wavelet analysis of EPR spectrum (Fig. 3). (b) Wavelet analysis of spectrum (Fig. 3) after eliminating the broad line. Horizontal and vertical axis denote $t$ and $s$, respectively.

scale of magnetic field $t_{1}$ equals $B_{1}$. At the bottom part of Fig. 4a additional features with small line width are visible. We can eliminate the broad line, using the parameters obtained previously $\left(t_{1}, s_{1}\right)$, and the amplitude calculated from the formula (Eq. (2.10)). Thus, the filtered spectrum is obtained and shown in Fig. 4b. Analysis of it shows two relatively narrow lines, whose parameters are $\left(t_{2}, s_{2}\right)$ and $\left(t_{3}, s_{3}\right)$, with $A_{2}$ and $A_{3}$, correspondingly. The set of the parameters resulting from the analysis is presented in Table.

An attempt has been made to synthesize the original EPR spectrum (Fig. 3) from the obtained parameters. The results are displayed in Fig. 5. The blue line, which is the final result of the analysis, can be compared to the plot in Fig. 3. It is the sum of the three extracted components: orange line - \#1, green line - \#2 and magenta - \#3.

One may conclude that the wavelet analysis is a complementary tool for spectra interpretation; the synthesized spectrum resembles the experimental ob- 
TABLE

Parameters of the wavelet-detected EPR lines.

\begin{tabular}{c|c|c|c}
\hline \hline Component & $A$ & $t$ & $s$ \\
\hline 1 & 37.58 & 334.69 & 1.02 \\
2 & 16.93 & 333.44 & 0.36 \\
3 & 25.06 & 335.04 & 0.23
\end{tabular}

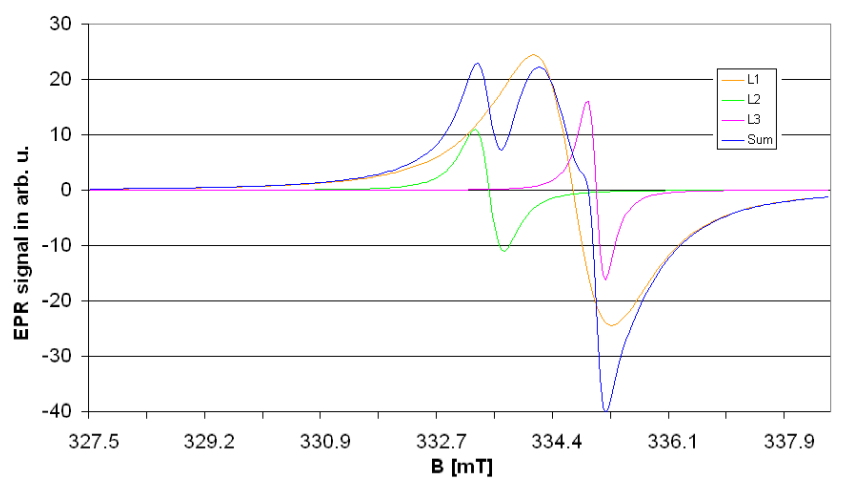

Fig. 5. Synthesis of EPR spectrum with the parameters shown in Table.

servation. As an additional utility one has the option of searching and eliminating unwanted features from the experimental data.

\section{References}

[1] B.J.B. Schein, E.C. Lingafelter, J.M. Stewart, J. Chem. Phys. 47, 5138 (1967).

[2] D.B. Percival, A.T. Walden, Wavelet Methods for Time Series Analysis, Cambridge University Press, Cambridge 2000.

[3] J.T. Białasiewicz, Wavelets and Approximations, Wydawnictwo Naukowo-Techniczne, Warszawa 2004, p. 66 (in Polish).

[4] P.B. Sczaniecki, B. Jakubaszek, L. Szczepańska, in: Magnetic Resonance and Related Phenomena, Vol. II, Extended Abstracts of the Joint 29th AMPERE 13th ISMAR Int. Conf., Eds. D. Ziessow, W. Lubitz, F. Lendzian, Technische Universität Berlin, Berlin 1998, p. 981. 